\title{
Development of Automatic Impedance Matching System for Resonant Cavity Applicator
}

\author{
YASUHIRO SHINDO ${ }^{*}$, KAZUO KATO ${ }^{2}$, HIDEAKI TAKAHASHI ${ }^{3}$, \\ TAKEO UZUKA ${ }^{4}$, YUKIHIKO FUJII ${ }^{4}$
}

\author{
${ }^{1}$ Graduate School of Science and Technology, and ${ }^{2}$ Department of Mechanical Engineering Informatics, Meiji \\ University, 1-1-1 Higashi-mita, Tama, Kawasaki, Kanagawa 214-8571, Japan \\ ${ }^{3}$ Niigata Cancer Center, Section of Neurosurgery, 2-15-3 Kawagishi-cho, Niigata 951-8566, Japan \\ ${ }^{4}$ Department of Neurosurgery, Brain Research Institute, Niigata University, 1-757 Asahimachi-dori, Niigata \\ 951-8585, Japan
}

\begin{abstract}
This paper describes a new system to match the impedance automatically for a re-entrant resonant cavity applicator during brain tumor hyperthermia treatments non-invasively. We have already discussed the effectiveness of the heating method using a manual impedance matching controller with heating experiments of an agar phantom and computer simulations. With this heating method, to generate heating power in the cavity, it is necessary to match the impedance and to tune the resonant frequency. In the initial prototype heating system, both were manually adjusted. However, it was difficult to match the impedance and to tune the frequency especially using a high resonant frequency.

To overcome this problem, we developed the automatic impedance matching system (AIMS). It is known that reflected power is generated when the impedance matching is not complete. In this system, to reduce the reflected power which is fed back to the computer, the stepping motor to turn the dial of the variable capacitors is controlled by developed software. To evaluate the developed AIMS, the heating experiments of the agar phantom, using several electromagnetic modes, were performed. The VSWRs are 1.05 and 1.01 using manual matching and AIMS, respectively. From these results, we found that the temperature rise in the agar phantom using AIMS was about $180 \%$ (with $\mathrm{TM}_{012}$-like mode) and about $110 \%$ (with $\mathrm{TM}_{010}$-like mode) more than using the manual type controller under the same heating condition. It was found that the proposed system was very effective for hyperthermia treatment using the resonant cavity applicator even when the resonant frequency was high.
\end{abstract}

Key Words: impedance matching, resonant cavity applicator, electromagnetic field patterns, non-invasive.

\section{Introduction}

As the human brain is protected by the skull, it is not easy to heat deep brain tumors non-invasively

Received 25 December, 2009, Accepted 18 April, 2010. *Corresponding author; Tel, +81-44-934-7355; Fax, +81-44-934-7907; e-mail, yshindo@isc.meiji.ac.jp

doi : $10.3191 /$ thermalmed.26.63

(C) 2010 Japanese Society for Thermal Medicine 
with electromagnetic energy for hyperthermia treatments. Several heating methods have been proposed to heat deep tumors. Some examples are radio frequency (RF) capacitive heating applicators ${ }^{1)}$, annular phased array applicators ${ }^{2}$, and coaxial transverse electromagnetic (TEM) applicators ${ }^{3)}$. Some of these methods have been in practical use. However, all of them have advantages and disadvantages, and a successful heating method has not yet been realized.

From 1989, a non-invasive heating method using a re-entrant type resonant cavity applicator was proposed and many positive results have been generated since then ${ }^{4,6-10)}$. These results show that this heating method is capable of heating deep-seated tumors non-invasively.

In the proposed heating system, different from other high-frequency heating systems ${ }^{5)}$, generating heating power inside the cavity requires not only impedance matching input and output, but also the tuning of the exciting frequency and the resonant frequency of the cavity. When the matching and tuning operations are completely performed, the electromagnetic field pattern is generated inside the cavity and the human tumors can be heated concentrically. From our current research, it is found that the resonant frequency can be estimated by computer simulations ${ }^{6-13)}$. So in this study, resonant frequencies of the cavity were calculated by the finite element method (FEM) and were used as the exciting frequencies. In the early prototype heating system, as a manual type impedance matching controller was used. It was difficult to match the impedance in high frequency electromagnetic modes (over $200 \mathrm{MHz}$ ) in this early stage. Especially when using $\mathrm{TM}_{012}$-like mode, we could not get a sufficient temperature rise in the hyperthermia treatment with the manual controller ${ }^{9)}$ To overcome this problem, we have developed an automatic impedance matching system (AIMS).

In this paper, we propose a new matching system which can adjust the impedance between input and output power automatically.

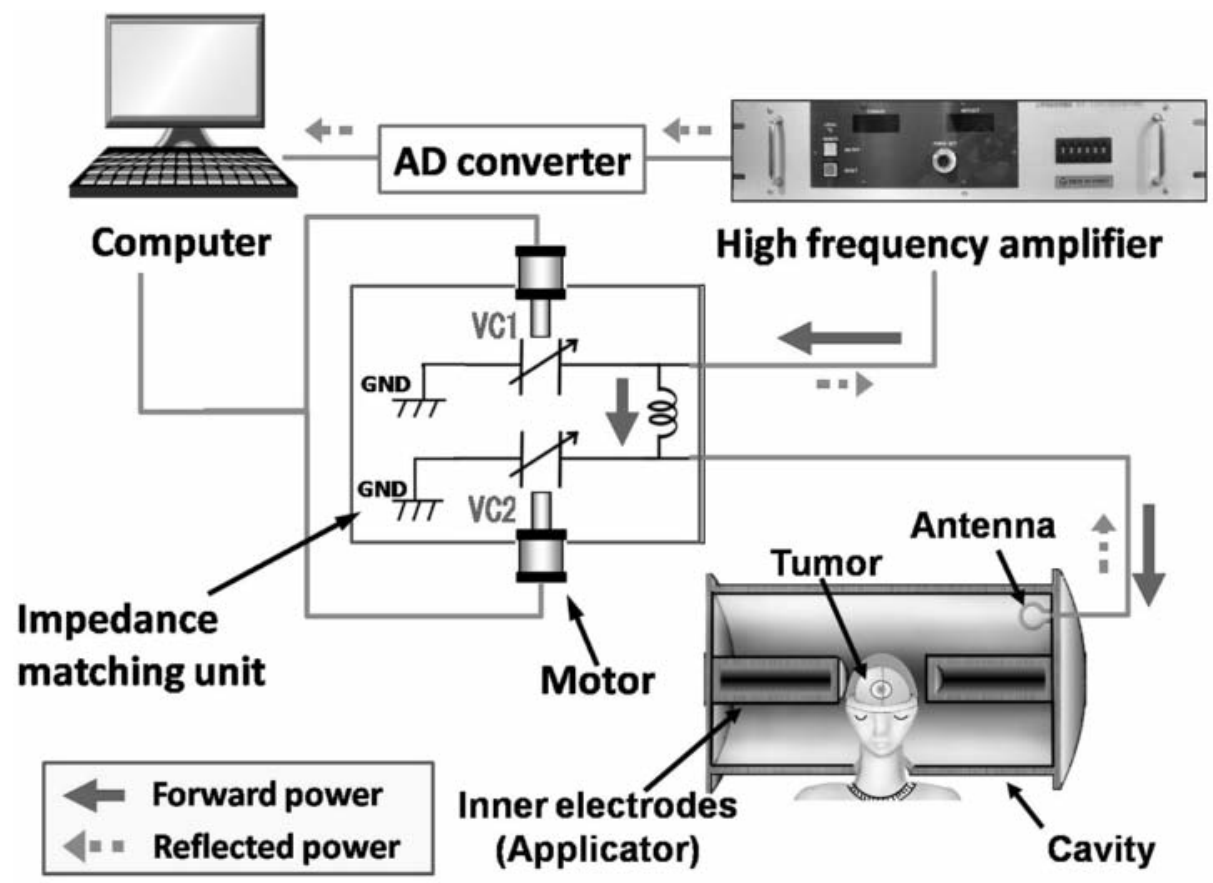

Fig. 1. Illustration of the automatic impedance matching system (AIMS). 
First, the developed matching system for a non-invasive heating system using the re-entrant type resonant cavity applicator is presented. This system feeds back the reflected power generated in the cavity to the impedance matching unit. When the impedance does not match, the unit decreases the reflected power until it matches. Second, the experimental results of heating agar phantoms using AIMS with several electromagnetic modes are discussed. Finally, from these results, it is shown that the developed system can accurately adjust the impedance automatically and is useful for non-invasive hyperthermia treatments.

\section{Methods and materials}

\section{Development of Automatic Impedance Matching System (AIMS)}

Fig. 1 shows an illustration of the automatic impedance matching system (AIMS). In Fig. 1, a human head is placed in the center of the inner electrodes, and is heated by electromagnetic fields inside the cavity without touching the human body with the applicator. The impedance matching unit is connected to the cavity, and the heating power is supplied by the high frequency amplifier. When the impedance does not match completely, the reflected power generated is fed back to the computer with the AD converter, and the motor drive unit connected with the impedance matching unit is controlled automatically to match the input and output impedance.

Fig. 2 shows a photograph of the developed system. It consists of a cavity, a high frequency amplifier, a motor drive unit, an AD converter, a computer, and an impedance matching unit.

Fig. 3 shows the cross-sectional view of the cavity. The cavity is made of an aluminum plate, and is $500 \mathrm{~mm}$ in diameter and $1000 \mathrm{~mm}$ in height. To concentrate the heating energy in the center of the cavity, inner electrodes of $100 \mathrm{~mm}$ in diameter and

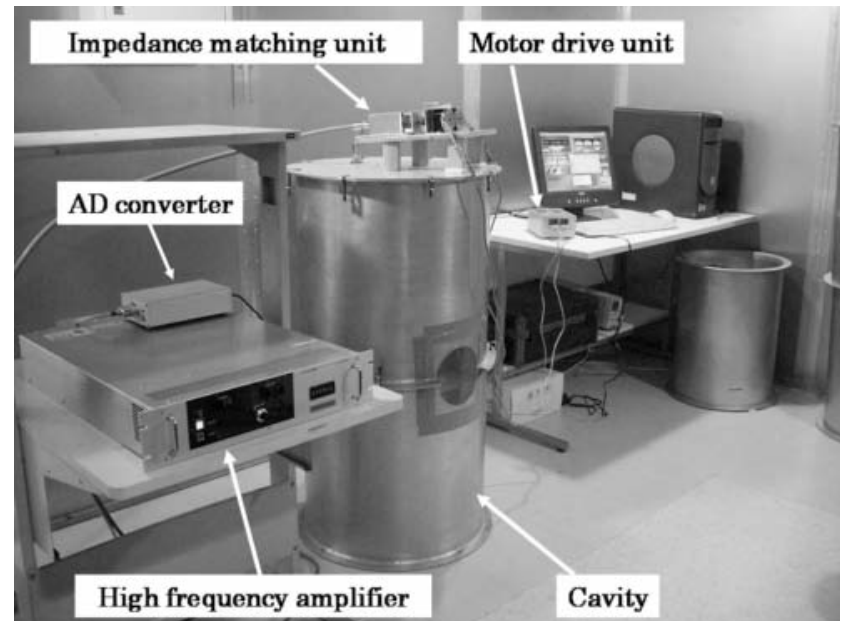

Fig. 2. Set up of the system.

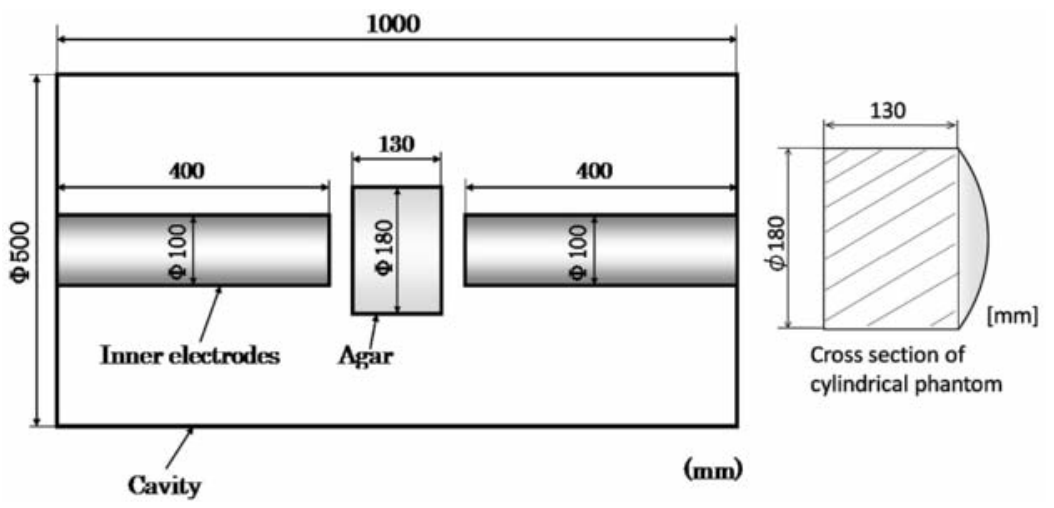

Fig. 3. Cross-sectional view of the cavity. 
$400 \mathrm{~mm}$ in height are used. We made the cylindrical agar phantom $180 \mathrm{~mm}$ in diameter and $130 \mathrm{~mm}$ in height for the heating experiments.

\section{Impedance matching unit in comparison with the manual proto type}

We used a manual type controller to match impedance in the prototype heating system ${ }^{6-9)}$. Fig. 4a shows the manual type impedance matching unit with two dials for variable capacitors (VC1 and VC2). Fig. 4b shows the circuit diagram for impedance matching. The adjustable ranges of the $\mathrm{VCl}$ and VC2 are from $20 \mathrm{pF}$ to $180 \mathrm{pF}$ and from $6 \mathrm{pF}$ to $80 \mathrm{pF}$ listed in Table I, respectively. In this automatic system, we developed a motor drive type impedance matching controller to match impedance more accurately. A photograph of the developed motor drive system is shown in Fig. 4c. The

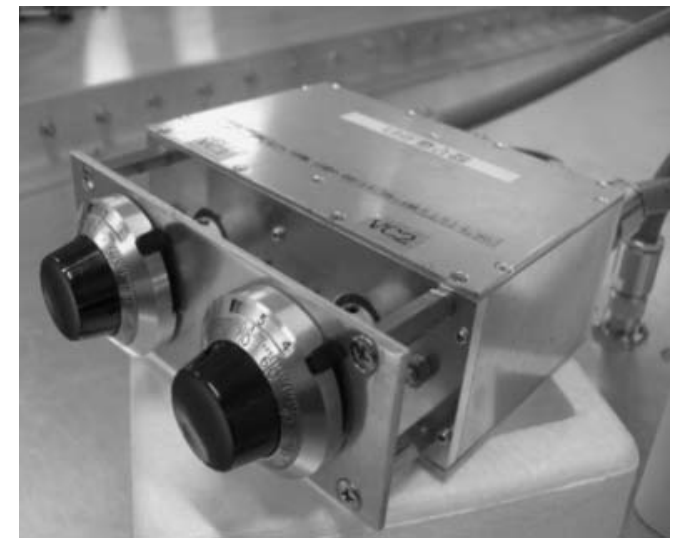

$\boldsymbol{a}$
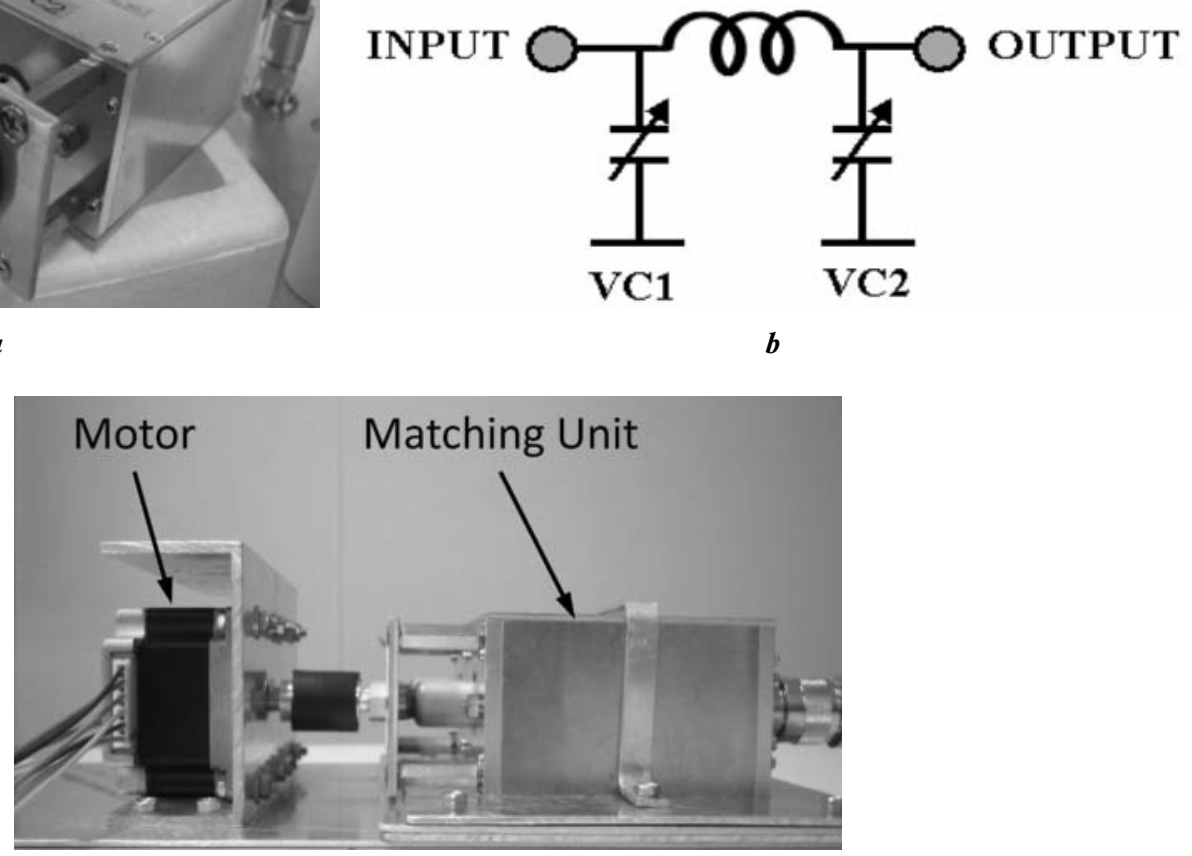

c

Fig. 4. Impedance matching unit. $\quad \boldsymbol{a}$, Manual type unit. $\quad \boldsymbol{b}$, Circuit diagram for impedance matching. c, Motor drive matching unit.

Table I. Adjustable ranges of the impedance matching unit.

\begin{tabular}{|c|c|c|}
\hline Dial $[\mathrm{rev}]$ & VC1 capacity $[\mathrm{pF}]$ & VC2 capacity $[\mathrm{pF}]$ \\
\hline 0 & 20 & 6 \\
\hline 19.9 & 180 & 80 \\
\hline
\end{tabular}


minimum adjustment angle for the rotation of the dials for the VC1 and VC2 used in the system is $1.8(\mathrm{deg} / \mathrm{step})$.

\section{Automatic control program}

Fig. 5 shows the procedure for the proposed automatic impedance matching operation. First, an initial value is set for VC2 and an "All range searching" is started. In order to measure the reflected power, the $\mathrm{VC} 1$ motor is rotated every 10 steps roughly over the full rotation angles. After the "All range searching", VC1 is set to around the point of minimum reflected power. Second, a "Detailed searching" was carried out. Furthermore, in order to find the minimum value of reflected power with more accuracy, VC1 is rotated by 1 step increment. When the reflected power decreases below an arbitrary threshold value, the rotation is stopped. Here, we set the threshold value to $0.1 \%$ of the heating power so that the voltage standing wave ratio (VSWR) of the AIMS becomes lower than that of the manual operation results. VC2 is also obtained as well as VC1. When the impedance matching operation was carried out by the AIMS, heating power was set to $8 \mathrm{~W}$. After matching the impedance automatically, we heated the agar phantom with $30 \mathrm{~W}$ of heating power.

The features of the AIMS are summarized below:

(1) During automatic impedance matching operations, changes in reflected wave power, input power and VSWRs are displayed. Therefore, the operator can operate the AIMS while checking these values so the operation does not terminate on a local minimum.

(2) The AIMS is in a state in which the prediction results of the SAR and temperature distributions calculated by the FEM are displayed, and impedance matching operations can be carried out.
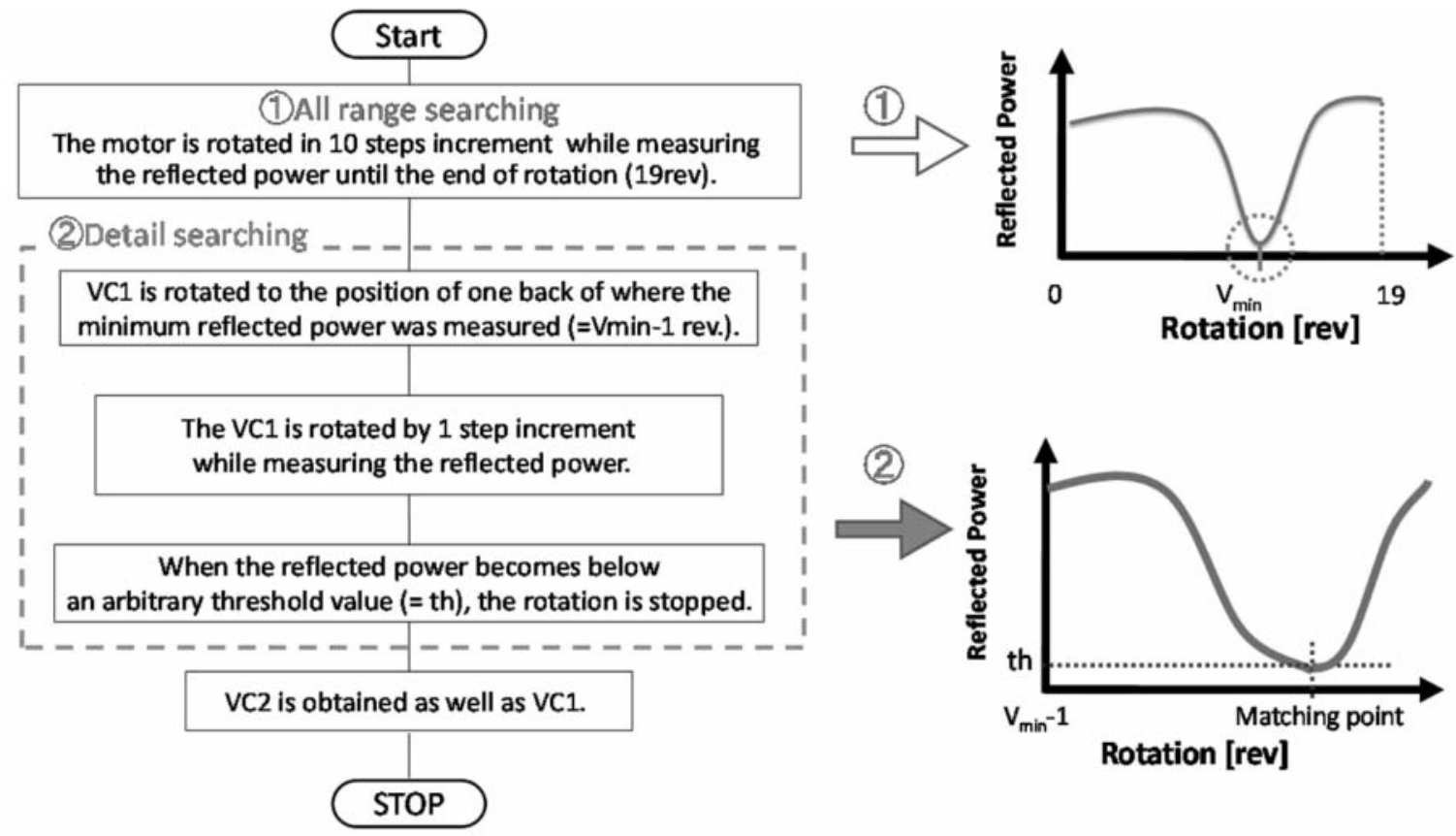

Fig. 5. Procedure for the proposed automatic impedance matching operation. 
(3) Changes of the measured temperature by the fiber-optic thermometer can be displayed, therefore the operator can operate the AIMS while checking for the target temperature.

(4) Since the AIMS is operated by remote control, the operator can work in a safe environment.

\section{Experimental conditions for heating a human head agar phantom with TM o10 $^{-l i k e}$ mode}

To evaluate the developed AIMS when using a dominant electromagnetic mode, the heating experiments of the human head agar phantom used a $\mathrm{TM}_{010}$-like mode in both matching methods.

Fig. 6 shows the set up of a human head agar phantom. The human head agar is $215 \mathrm{~mm}$ horizontally and $235 \mathrm{~mm}$ vertically ${ }^{13)}$. In order to heat the center of the brain, the human head agar is placed so that the distance from the cavity wall to the top of the head is $270 \mathrm{~mm}$.

The experimental heating conditions are listed in Table II. In this experiment, heating power is $30 \mathrm{~W}$, heating time is $30 \mathrm{~min}$, and both resonant frequencies are $144.00 \mathrm{MHz}$.

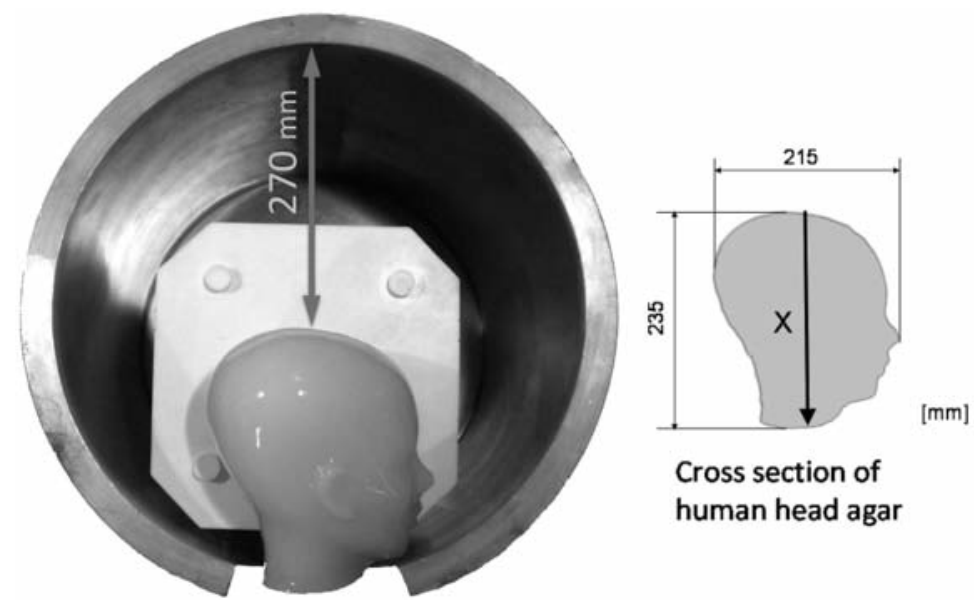

Fig. 6. Set up of the human head agar phantom.

Table II. Experimental conditions.

\begin{tabular}{|c|c|c|c|c|}
\hline & Heating power & Heating time & Resonant frequency & Impedance matching \\
\hline (a) & $30 \mathrm{~W}$ & $30 \mathrm{~min}$ & $144.00 \mathrm{MHz}$ & Manual \\
\hline (b) & $30 \mathrm{~W}$ & $30 \mathrm{~min}$ & $144.00 \mathrm{MHz}$ & AIMS \\
\hline
\end{tabular}

\section{Results}

Evaluation of Automatic Impedance Matching System (AIMS) with manual matching at TM o12- $^{-}$ like mode

To evaluate developed AIMS, heating experiments using an agar phantom were performed. Here, we compare the two results of impedance matching using the manual system and the developed automatic system (AIMS). When the manual matching is finished, the reflected power is $0.8 \mathrm{~W}$ and VSWR is 1.055 . 
The operating times of the AIMS and manual matching operations are about $30 \mathrm{~min}$ and $60 \mathrm{~min}$, respectively.

Fig. 7 shows the measured data for the reflected power under the matching operation using the AIMS when VC1 matches. In Fig. 7a, after an "All range searching", we can see that the minimum value of reflected power exists between $\mathrm{VC} 1=13$ and $\mathrm{VC} 1=15$ rev. In Fig. 7b, after a "Detailed searching", it can be seen that the matching value for $\mathrm{VC} 1$ is $13.58 \mathrm{rev}$ when the reflected power reaches its minimum value $(0.187 \mathrm{~W}, \mathrm{VSWR}=1.012)$. We can determine the value of VC2 in the same way.

Fig. 8 shows the measured reflected power when VC2 matches. Fig. $8 \mathrm{~b}$ shows the corresponding value for VC2 $(0.01 \mathrm{rev})$ when the impedance matches.

Comparison of heating results by manual impedance matching and by the AIMS at TM o12 $_{2}-$ like mode

Fig. 9 shows the thermal images of a sagittal slice of the agar phantom. The thermal images were taken by an infrared thermal camera (NEC SANEI TH7102WX) after 60 min of heating using the re-entrant resonant cavity. Both of the developed AIMS and the manual type impedance matching
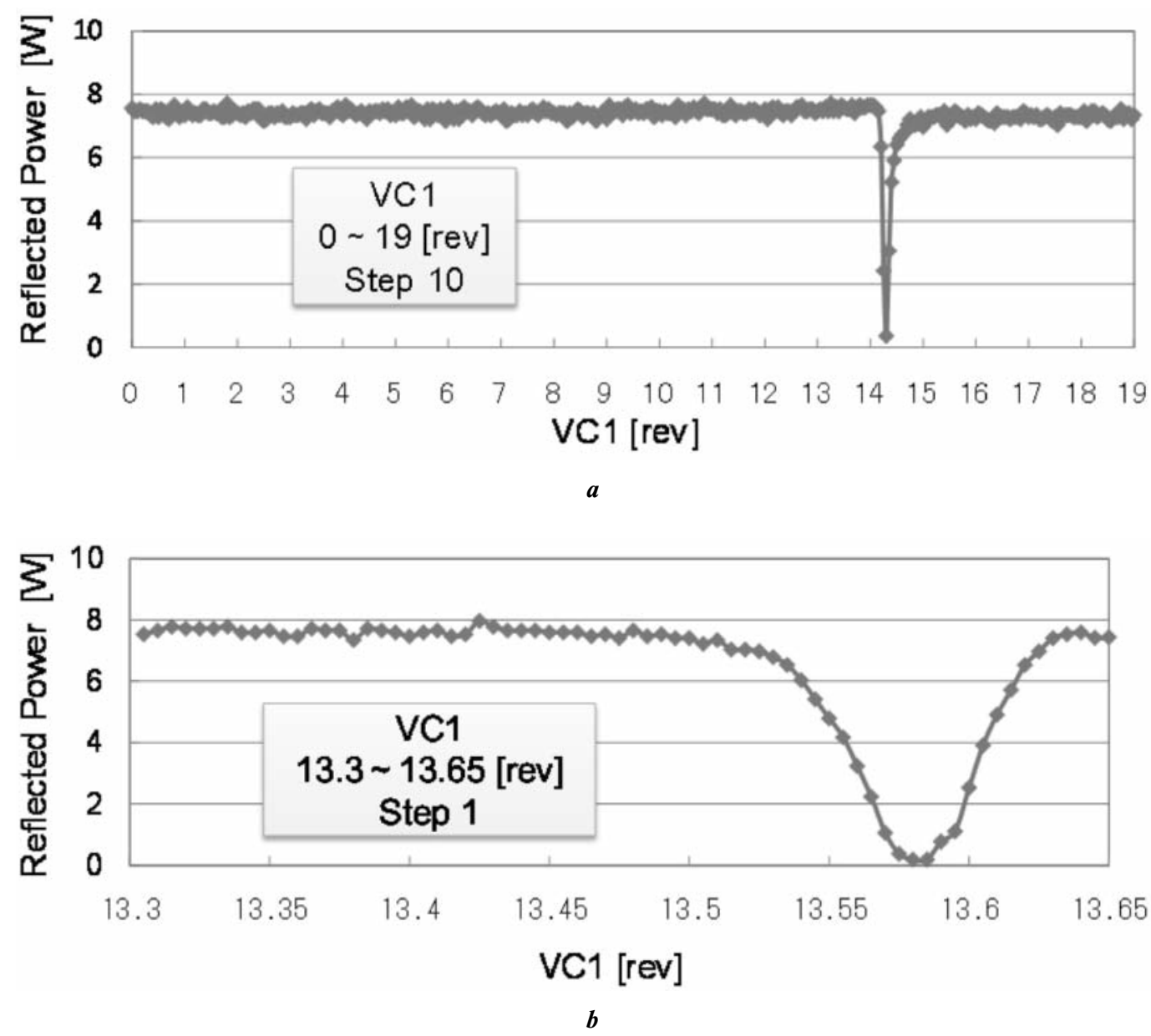

Fig. 7. Reflected power data of VC1. a, VC1 all range searching. b, VCl detailed searching. 
controllers were examined ${ }^{6-9)}$. Experimental heating conditions are listed in Table III. From Fig. 9, both of the thermal images show that the center of the agar phantom was heated to a maximum temperature without physical contact. However, the temperature increase using the AIMS was greater than that produced when using the manual impedance matching controller. It can be seen that the developed AIMS is useful for the re-entrant type resonant cavity applicator.
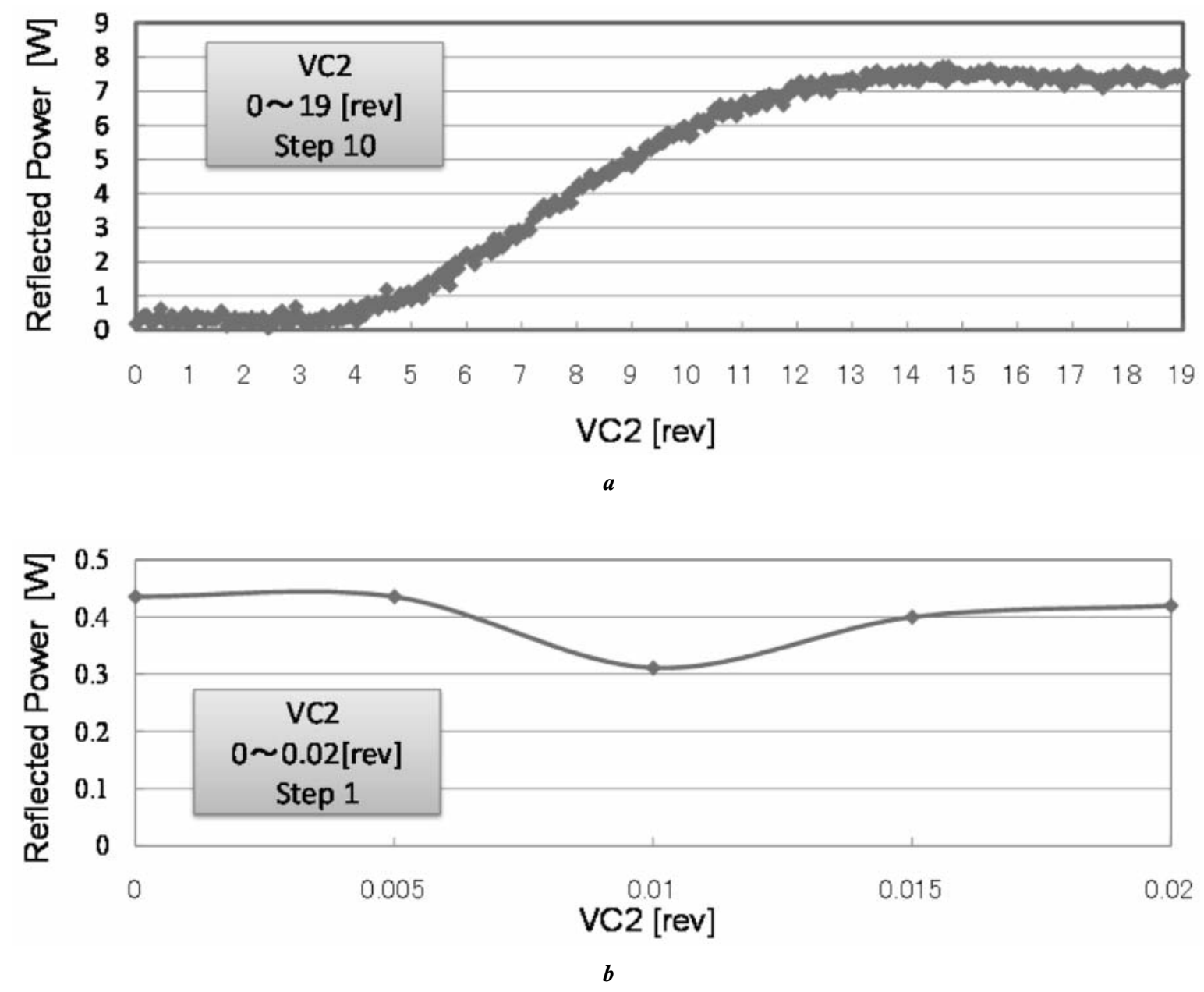

Fig. 8. Reflected power data of VC2. $\boldsymbol{a}, \mathrm{VC} 2$ all range searching. $\boldsymbol{b}, \mathrm{VC} 2$ detailed searching.

Table III. Experimental conditions.

\begin{tabular}{|c|c|c|c|c|}
\hline & Heating power & Heating time & Resonant frequency & Impedance matching \\
\hline (a) & $30 \mathrm{~W}$ & $60 \mathrm{~min}$ & $377.55 \mathrm{MHz}$ & Manual \\
\hline (b) & $30 \mathrm{~W}$ & $60 \mathrm{~min}$ & $376.22 \mathrm{MHz}$ & AIMS \\
\hline
\end{tabular}




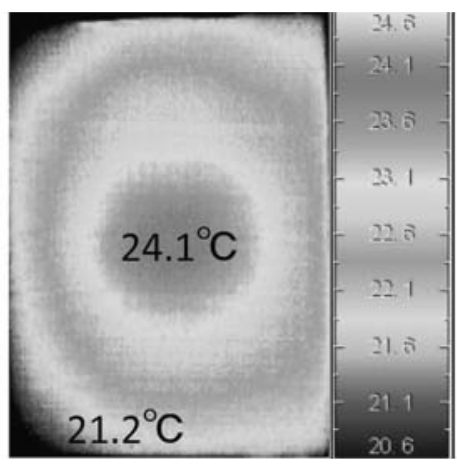

$a$

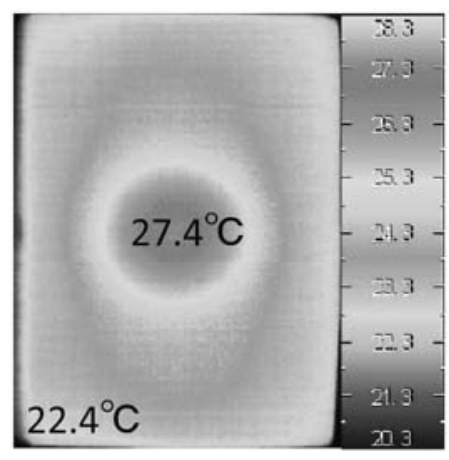

$b$

Fig. 9. Thermal images. a. Manual matching. b. Using the AIMS.

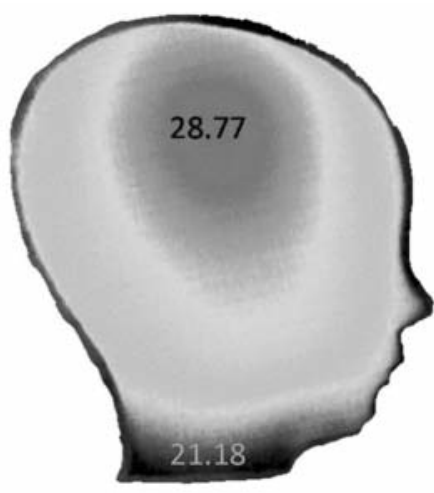

$a$

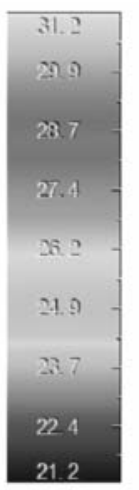

21.2

Fig. 10. Thermal images of the human head agar phantom ( $\mathrm{f}=144.0 \mathrm{MHz}$ ) $\boldsymbol{a}$, Manual matching. $\boldsymbol{b}$, Using the AIMS.

\section{Comparison of heating results of human head type agar phantom using the manual impedance}

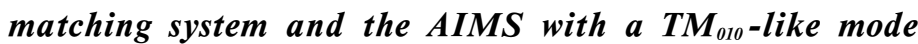

Fig. 10 shows the thermal images of human head agar phantom. They are taken by an infrared thermal camera. Both of the results show that the center of the brain is heated to a maximum temperature without heating the neighboring eyeballs and nose. Furthermore, with an attention to the temperature rise, Fig. 10 shows an $8.6^{\circ} \mathrm{C}$ increase in the AIMS and a $7.6^{\circ} \mathrm{C}$ increase in the manual impedance matching system. By using the AIMS, the temperature increased more, by approximately $1^{\circ} \mathrm{C}$, in comparison to the manual matching system.

\section{Discussion}

Interstitial hyperthermia has been used in clinical practice to invasively heat deep tumors ${ }^{14,15)}$. However, this method has both advantages and disadvantages. One of the advantages is that direct and local heating of the area around the needle is possible. Disadvantages are that this heating method has a small heating area and is an invasive heating method. In the developed prototype heating system using a resonant cavity to non-invasively heat deep tumors, the matching operations were performed manually. 
If the impedance matching in the heating system with a resonant cavity is not complete, most of the microwave power will be reflected and therefore the temperature inside a targeted object will not increase.

It is not easy to completely match the impedance in the prototype heating system using the manual impedance matching controller. As shown in Figs. 7 and 8, impedance matching ranges for $\mathrm{VC} 1$ and VC2 are very narrow, almost all of the heating power is reflected except for these corresponding matching ranges of $\mathrm{VC} 1$ and $\mathrm{VC} 2$. It was almost impossible to manually rotate VC1 and VC2 per 0.01 rev to match the impedance. It is found that the proposed heating method, which uses the re-entrant type resonant cavity applicator with the developed AIMS is useful for deep tumor hyperthermia treatments.

As shown in Fig. 11, when comparing the temperature rise of the AIMS method to the manual

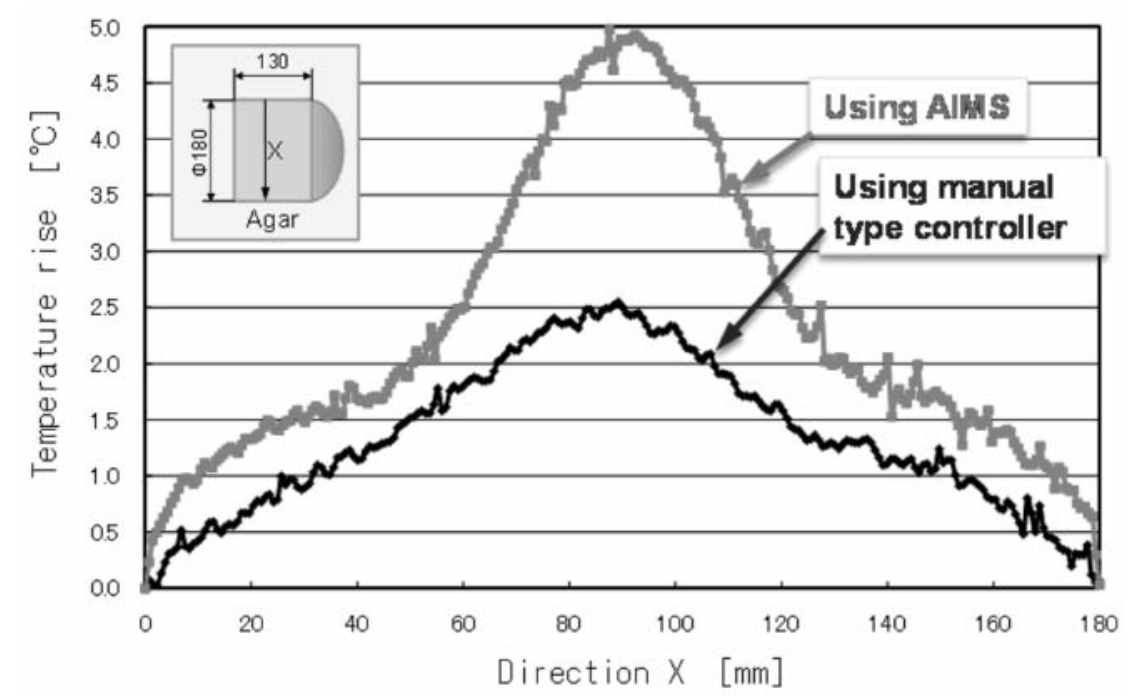

$a$

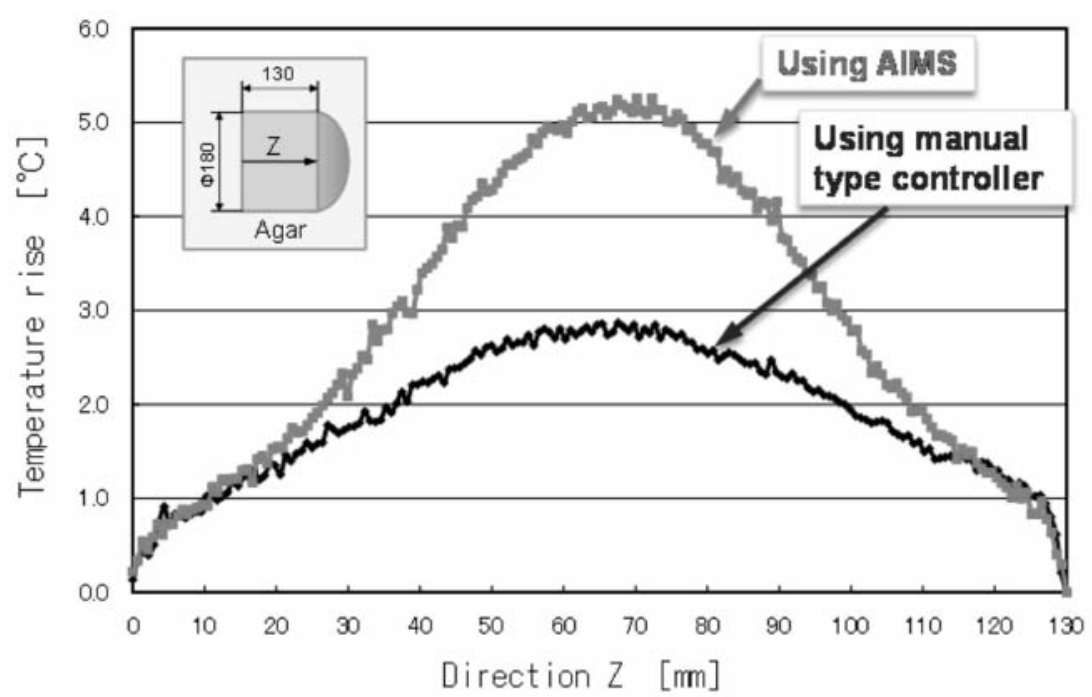

$\boldsymbol{b}$

Fig. 11. Temperature rise profiles. $\boldsymbol{a}$, On the $\mathrm{x}$-axis. $\boldsymbol{b}$, On the $\mathrm{z}$-axis. 


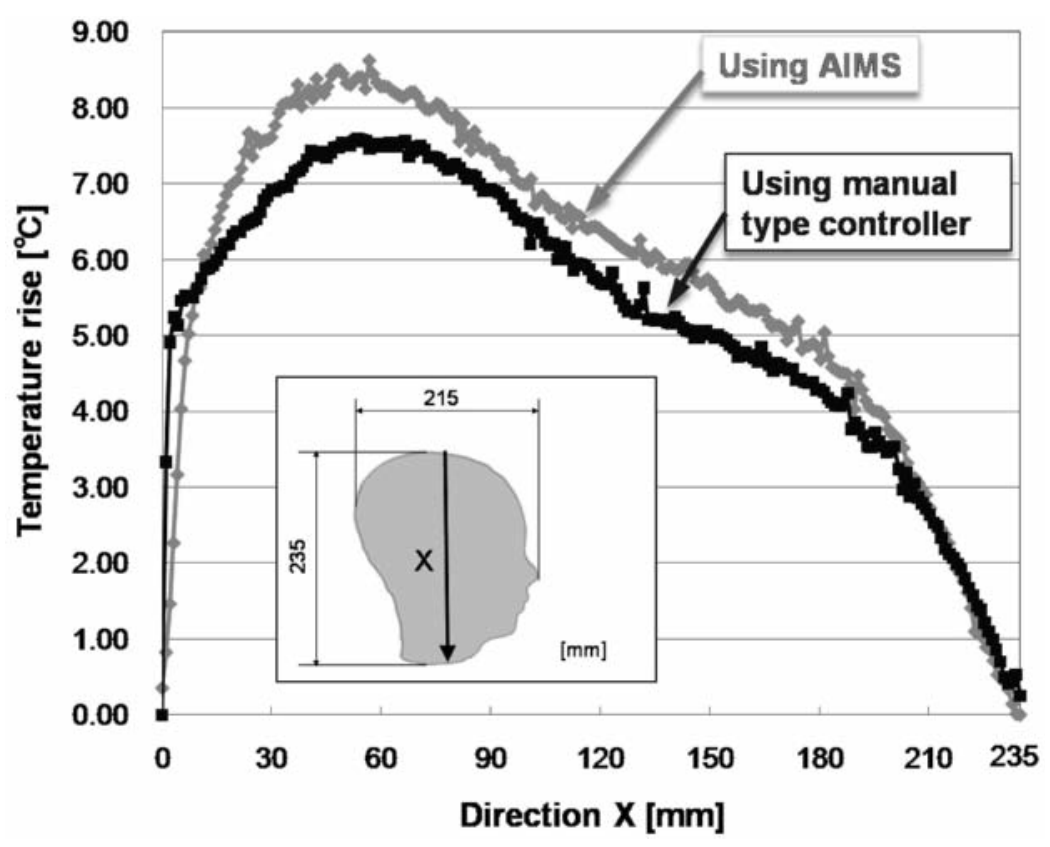

Fig. 12. Temperature profiles of the human head agar phantom (on the $\mathrm{x}$-axis).

method using a manual impedance controller at $\mathrm{TM}_{012}$-like mode ${ }^{12)}$, the temperature rise of the AIMS method is greater than that of the heating results using the manual impedance matching controller. The AIMS temperature rise is approximately twice that of the manual method.

Furthermore, Fig. 12 shows the temperature profiles of the human head agar phantom. From Fig. 12, the developed AIMS is effective even in the dominant electromagnetic mode ( $\mathrm{TM}_{010}$-like mode).

From these results, we can see that it is possible to automatically match impedance with high accuracy by using the developed AIMS.

\section{Conclusion}

We proposed the automatic impedance matching system for the developed re-entrant resonant applicator to non-invasively heat deep brain tumors.

From the experimental results, it is confirmed that the proposed AIMS is applicable to successful hyperthermia treatments.

We are now trying to develop a high speed automatic impedance matching system which contains a complete automatic tuning system consisting of both impedance matching and frequency tuning controllers. In order to confirm the safety of organic tissue within the head such as an eyeball, estimation of temperature distributions using a 3-D human head model reconstructed from 2-D medical images and animal experiments will be performed. 


\section{References}

1) Nussbaum G.H., Sidi J., Rouhanizadeh N., Morei P., Jasmin C., Convert G., Mabire J.P., Azam G. : Manipulation of central axis heating patterns with a prototype, three-electrode capacitive device for deep-tumor hyperthermia. IEEE Trans Microwave Theory Tech, 33 : 620-625, 1986.

2) Turner P.F.: Regional hyperthermia with an annular phased array. IEEE Trans Biomed Eng, 31: 106-114, 1984.

3) Langendijk J.J.W.: A new coaxial TEM radiofrequency/microwave applicator for non-invasive deep-body hyperthermia. J Microwave Power, 18 : 367-375, 1983.

4) Matsuda J., Kato K., Saitoh Y.: The application of a re-entrant type resonant cavity applicator to deep and concentrated hyperthermia. Jpn J Hyperthermic Oncol, 4 : 111-118, 1988. (Japanese)

5) Tezuka M., Mizusawa M., Noguchi K., Betsudan S., Katagi T., Imanishi Y. : A matching circuit in automatic matching system for antennas. "The transactions of the Institute of Electronics, Information and Communication Engineers”. 85, pp.1977-1980, 2002.

6) Yabuhara T., Kato K. : Design and construction of resonant cavity applicator for brain tumor hyperthermia treatment by using electromagnetic modes without contact. "Journal of Japan Society for Design Engineering”. 44, pp.236-245, 2009.

7) Kato K., Matsuda J., Saitoh Y.: A re-entrant type resonant cavity applicator for deep-seated hyperthermia treatment. "Proc. of Annual International Conf. of IEEE EMBS vol. 11". pp.1712-1713, 1989.

8) Yabuhara T., Kato K., Tsuchiya K., Shigihara T., Shindo Y., Iwazaki R., Uzuka T., Fujii Y., Takahashi H. : Finite element analysis of the re-entrant type resonant cavity applicator for brain tumor hyperthermia, "Proc. of $29^{\text {th }}$ Annual International Conference of the IEEE EMBS”. pp.3540-3543, 2007.

9) Shindo Y., Kato K., Tsuchiya K., Shigihara T., Iwazaki R., Uzuka T., Tkahashi H., Fujii Y.: Heating properties of re-entrant resonant applicator for brain tumor by electromagnetic heating modes. "Proc. of $29^{\text {th }}$ Annual International Conference of the IEEE EMBS". pp.3609-3612, 2007.

10) Shindo Y., Kato K., Hirashima T. and Yabuhara T.: Development of automatic impedance matching system for hyperthermia treatment using resonant cavity applicator, "Proc. of $30^{\text {th }}$ Annual International IEEE EMBS Conference". pp.4376-4379, 2008.

11) Hirashima T., Kato K., Shindo Y., Iwazaki R., Yabuhara T., Uzuka T., Takahashi H., Fujii Y.: Heating properties of re-entrant resonant cavity applicator for brain tumor with simple head model, "Proc. of $30^{\text {th }}$ Annual International IEEE EMBS Conference”. pp.234-237, 2008.

12) Yabuhara T., Shindo Y., Kazuo K., Takahashi H., Uzuka T., Fujii Y.: Heating properties of resonant cavity applicator for brain tumor hyperthermia: TM-like modes permit heat production without physical contact. Thermal Med, 24 : 141-152, 2008.

13) Suzuki M., Kato K., Hirashima T., Shindo Y., Uzuka Y., Takahashi H. and Fujii Y.: Heating properties of the re-entrant type cavity applicator for brain tumor with several resonant frequencies., "Proc. of $31^{\text {st }}$ Annual International Conference of the IEEE EMBS". pp.3064-3067, 2009.

14) Emami B., Scott C., Perez C.A., Asbell S., Swift P., Grigsby P., Montesana A., Rubin P., Curan W., Derowe J., Aratu H., Fu K., Mores E. : Phase III study of interstitial thermoradiotherapy compared with interstitial radiotherapy alone in the treatment or recurrent or persistent human tumors. Int J Radiat Oncol Biol Phys, 34 : $1097-1104,1996$.

15) Saito K., Yoshimura H., Ito K., Aoyagi Y., Horita H. : Clinical trials of interstitial microwave hyperthermia by use of coaxial slot-antennas with two slots. IEEE Trans Microwave theory Tech, 52 : 1987-1991, 2004. 